\title{
ON THE GENERALIZED FENG-RAO NUMBERS OF NUMERICAL SEMIGROUPS GENERATED BY INTERVALS
}

\author{
M. DELGADO, J. I. FARRÁN, P. A. GARCÍA-SÁNCHEZ, AND D. LLENA
}

ABSTRACT. We give some general results concerning the computation of the generalized Feng-Rao numbers of numerical semigroups. In the case of a numerical semigroup generated by an interval, a formula for the $r^{\text {th }}$ Feng-Rao number is obtained.

\section{INTRODUCTION}

The Feng-Rao distance for a numerical semigroup was introduced in coding theory as a lower bound for the minimum distance of a one-point algebraic geometry (error-correcting) code (see [8]). This order bound, computed from Weierstrass semigroups, improves the lower bound for the minimum distance given by Goppa with the aid of the Riemann-Roch theorem. Moreover, the Feng-Rao distance is essential in a majority voting decoding procedure, which is the most efficient one for such types of codes (see [11]).

Even though the Feng-Rao distance was introduced for Weierstrass semigroups and for decoding purposes, it is just a combinatorial concept that makes sense for arbitrary numerical semigroups. This problem has been broadly studied in the literature for different types of semigroups (see [2], 3] or [12]). In numerical terms, the above mentioned improvement of the Goppa distance in coding theory means the following: For a semigroup $S$ with genus $g$ and $m \in S$ the Feng-Rao distance satisfies

$$
\delta(m+1) \geq m+2-2 g
$$

if $m>2 g-2$, and equality holds for $m>>0$.

On the other hand, the concept of minimum distance for an error-correcting code has been generalized to the so-called generalized Hamming weights. They were introduced independently by Helleseth et al. in [10] and Wei in [14 for applications in coding theory and cryptography, respectively.

The natural generalization of the Feng-Rao distance to higher weights was introduced in [9]. The computation of these generalized Feng-Rao distances turns out to

Received by the editor May 19, 2011 and, in revised form, November 22, 2011.

2010 Mathematics Subject Classification. Primary 20M14, 11Y55, 11 T71.

Key words and phrases. AG codes, weight hierarchy, numerical semigroups, order bounds, Goppa-like bounds, Feng-Rao numbers.

The first author was partially funded by the European Regional Development Fund through the program COMPETE and by the Portuguese Government through the FCT - Fundação para a Ciência e a Tecnologia under the project PEst-C/MAT/UI0144/2011.

The second author was supported by the project MICINN-MTM-2007-64704.

The third and fourth authors were supported by the projects MTM2010-15595, FQM-343 and FEDER funds.

The third author was also supported by the project FQM-5849. 
be a very hard problem. Actually, very few results are known about this subject, and they are completely scattered in the literature (see for example [1, 9] or [7]).

This paper studies the asymptotical behaviour of the generalized Feng-Rao distances, that is, $\delta^{r}(m)$ for $r \geq 2$ and $m>>0$. In fact, it was proven in [7] that

$$
\delta^{r}(m)=m+1-2 g+\mathrm{E}(S, r)
$$

for $m>>0$ (details in the next section). The number $\mathrm{E}(S, r)$ is called the $r$ th Feng-Rao number of the semigroup $S$, and is unknown except for a very few semigroups and concrete $r$ 's. For example, it was proven in [6] that

$$
\mathrm{E}(S, r)=\rho_{r}
$$

for hyperelliptic semigroups $S=\langle 2,2 g+1\rangle$, with multiplicity 2 and genus $g$, and for Hermitian-like semigroups $S=\langle a, a+1\rangle$, where $S=\left\{\rho_{1}=0<\rho_{2}<\cdots\right\}$. In fact, it is not even known yet if this formula holds for arbitrary numerical semigroups generated by two elements $S=\langle a, b\rangle$. Nevertheless, our experimental results point in this direction.

The main purpose of this paper is precisely to compute $\mathrm{E}(S, r)$ for semigroups generated by intervals, as a certain generalization of the Hermitian-like case. As a byproduct, we provide some general algorithms, implemented in GAP [5], to compute Feng-Rao numbers.

The paper is written as follows. Section 2 presents the general definitions concerning numerical semigroups, Feng-Rao distances and Feng-Rao numbers, and some convenient visualizations of integers for a given semigroup. The reader may find it useful to see some images in Subsection 2.3 .

The concept of amenable subset of a numerical semigroup is introduced in Section 3. It consists of a set that is closed for taking divisors. It implies that distances between elements are somehow controlled. Amenable sets play a fundamental role in some general results on Feng-Rao numbers of numerical semigroups. These results allowed the implementation of a function to compute the Feng-Rao numbers of a numerical semigroup which works quite well. It uses some of the functionalities of the GAP package numericalsgps [5] and will hopefully be part of a future release of that package. We give in this way some general results. Among them, an important lemma shows that the divisors of a configuration are the divisors of the shadow plus the elements above the ground.

Many examples computed with the referred function helped us to gain the necessary intuition to obtain a formula for the $r^{\text {th }}$ Feng-Rao number of a numerical semigroup generated by an interval, which is presented in Section 4 . This is the last and main result of this paper, and we briefly explain it in the sequel.

Recall that we are aiming to find a formula for $\delta^{r}(m)$, when $S$ is a semigroup generated by an interval of integers. The strategy will be as follows: Suppose that there is an amenable set $M$ which is an optimal configuration whose shadow $L_{M}=$ $[m, m+a+b) \cap M$ does not contain the ground (that is, $L_{M} \neq[m, m+a+b) \cap \mathbb{N}$ ). Then, using the results of Subsection 4.2, we can construct an $r$-amenable set $N$ (said to be ordered amenable) whose shadow $L_{N}$ is an interval starting in $m$ and has no more elements than $\sharp L_{M}$. Furthermore, by Proposition 28 , $\sharp \mathrm{D}\left(L_{N}\right) \leq \sharp \mathrm{D}\left(L_{M}\right)$, which implies that the number of divisors of $N$ is no bigger than the number of divisors of $M$ and therefore $N$ is also an optimal configuration. It follows that ordered amenable sets are optimal configurations. Thus, the problem of computing the generalized Feng-Rao numbers is reduced to counting the divisors of intervals 
of the form $[m, m+\ell] \cap \mathbb{N}$, with $\ell \leq a+b-1$. This is done by Corollary 24. The main result, which gives a formula, then follows.

\section{Definitions AND BASIC RESUlts}

This section is divided into several subsections. We start with several basic definitions and we introduce some notation. The reader is referred to the book 13 . for details. Then we give the definition of generalized Feng-Rao numbers and end the section by giving a way to visualize the integers which is convenient for our purposes.

2.1. Basic definitions and notation. Let $S$ be a numerical semigroup, that is, a submonoid of $\mathbb{N}$ such that $\sharp(\mathbb{N} \backslash S)<\infty$ and $0 \in S$. Denote, respectively, by $g:=\sharp(\mathbb{N} \backslash S)$ and $c \in S$ the genus and the conductor of $S$, being $c$ by definition the (unique) element in $S$ such that $c-1 \notin S$ and $c+l \in S$ for all $l \in \mathbb{N}$. Note that if $S$ is the Weierstrass semigroup of a curve $\chi$ at a point $P$, then $g$ is equal to the geometric genus of $\chi$, and the elements of $G(S):=\mathbb{N} \backslash S$ are called the Weierstrass gaps at $P$. For an arbitrary semigroup, these elements are simply called gaps.

It is well known (see for instance [13, Lemma 2.14]) that $c \leq 2 g$, and thus the "largest gap" of $S$ is $c-1 \leq 2 g-1$. The number $c-1$ is precisely the Frobenius number of $S$. The multiplicity of a numerical semigroup is the least positive integer belonging to it.

We say that a numerical semigroup $S$ is generated by a set of elements $G \subseteq S$ if every element $x \in S$ can be written as a linear combination,

$$
x=\sum_{g \in G} \lambda_{g} g,
$$

where finitely many $\lambda_{g} \in \mathbb{N}$ are non-zero. In fact, it is classically known that every numerical semigroup is finitely generated, that is, we can find a finite set $G$ generating $S$. Furthermore, every generator set contains the set of irreducible elements, $x \in S$ being irreducible if $x=u+v$ and $u, v \in S$ implies $u \cdot v=0$, and this set actually generates $S$, so that it is usually called "the" generator set of $S$, whose cardinality is called embedding dimension of $S$ (more details in [13]). Most of the time, we will suppose $S$ is minimally generated by $\left\{n_{1}<\cdots<n_{e}\right\}$. Its embedding dimension is $e$. Note that if $a$ and $b$ are integers, with $b<a$, and $S$ is minimally generated by the interval $[a, a+b] \cap \mathbb{N}$, then $n_{1}$ is $a, n_{e}-n_{1}$ is $b$ and the embedding dimension is $b+1$.

Finally, if we enumerate the elements of $S$ in increasing order,

$$
S=\left\{\rho_{1}=0<\rho_{2}<\cdots\right\},
$$

we note that every $x \geq c$ is the $(x+1-g)^{\text {th }}$ element of $S$, that is, $x=\rho_{x+1-g}$.

The last part of this paper will be devoted to semigroups generated by intervals.

Let $a$ be a positive integer and $b$ an integer with $0<b<a$. Let $S=\langle a, a+$ $1, \ldots, a+b\rangle$.

2.2. Feng-Rao numbers. Next we introduce the definitions for generalized FengRao distances. Although there is a subsection dedicated to the concept of divisor, we already need the definition. 
Definition 1. Given $x \in S$, we say that $\alpha \in S$ divides $x$ if $x-\alpha \in S$. We denote by $\mathrm{D}(x)=\{\alpha \in S \mid x-\alpha \in S\}$ the set of divisors of $x$.

Definition 2. Let $S$ be a numerical semigroup. For $m_{1} \in S$, let $\nu\left(m_{1}\right):=\sharp \mathrm{D}\left(m_{1}\right)$. The (classical) Feng-Rao distance of $S$ is defined by the function

$$
\begin{aligned}
\delta_{F R}: S & \longrightarrow \mathbb{N} \\
m & \mapsto \delta_{F R}(m):=\min \left\{\nu\left(m_{1}\right) \mid m_{1} \geq m, \quad m_{1} \in S\right\} .
\end{aligned}
$$

There are some well-known facts about the functions $\nu$ and $\delta_{F R}$ for an arbitrary semigroup $S$ (see 11, 12, or 2] for further details). An important one is that $\delta_{F R}(m) \geq m+1-2 g$ for all $m \in S$ with $m \geq c$, and that equality holds if, moreover, $m \geq 2 c-1$ (see also Proposition 7).

We will simplify the notation by writing $\delta(m)$ for $\delta_{F R}(m)$.

The classical Feng-Rao distance corresponds to $r=1$ in the following definition.

Definition 3. Let $S$ be a numerical semigroup, $m \in S$ and $r \geq 1$.

(a) A set $M \subset S \cap[m, \infty)$ with cardinality $r$ is called a $(S, m, r)$-configuration, or simply a configuration. For any such configuration $M=\left\{m_{1}, \ldots, m_{r}\right\} \subset S$, we will assume that $m \leq m_{1}<\cdots<m_{r}$.

(b) Let $\mathrm{D}(M):=\mathrm{D}\left(m_{1}\right) \cup \cdots \cup \mathrm{D}\left(m_{r}\right)$ (we will also write $\mathrm{D}\left(m_{1}, \ldots, m_{r}\right)$ for $\mathrm{D}(M)$; we may find the notation $\nu\left(m_{1}, \ldots, m_{r}\right):=\sharp \mathrm{D}\left(m_{1}, \ldots, m_{r}\right)$ in the literature).

The $r^{\text {th }}$ Feng-Rao distance of $S$ is defined by the function

$$
\begin{aligned}
\delta^{r}: S & \longrightarrow \mathbb{N} \\
m & \mapsto \delta^{r}(m)=\min \{\sharp \mathrm{D}(M) \mid M \text { is a }(S, m, r) \text {-configuration }\} .
\end{aligned}
$$

(c) A configuration $M$ with cardinality $r$ is said to be optimal if $\delta^{r}(m)=$ $\sharp \mathrm{D}(M)$.

Very few results are known for the numbers $\delta^{r}$, and their computation is very hard from both a theoretical and computational point of view. The main result we need describes the asymptotical behaviour for $m>>0$, and was proven in [7. This result tells us that there exists a certain constant $\mathrm{E}(S, r)$, depending on $r$ and $S$, such that

$$
\delta^{r}(m)=m+1-2 g+\mathrm{E}(S, r)
$$

for $m \geq 2 c-1$.

Definition 4. This constant $\mathrm{E}(S, r)$ is called the $r^{\text {th }}$ Feng-Rao number of the semigroup $S$. 7]).

Furthermore, it is also true that $\delta^{r}(m) \geq m+1-2 g+\mathrm{E}(S, r)$ for $m \geq c$ (see

Note that, for any non-negative integer $k$ and $m \geq 2 c-1, \delta^{r}(m+k)=k+\delta^{r}(m)$.

2.3. A convenient visualisation of the integers. We can think of the integers as points disposed regularly on a cylindrical helix (Figure 1). 


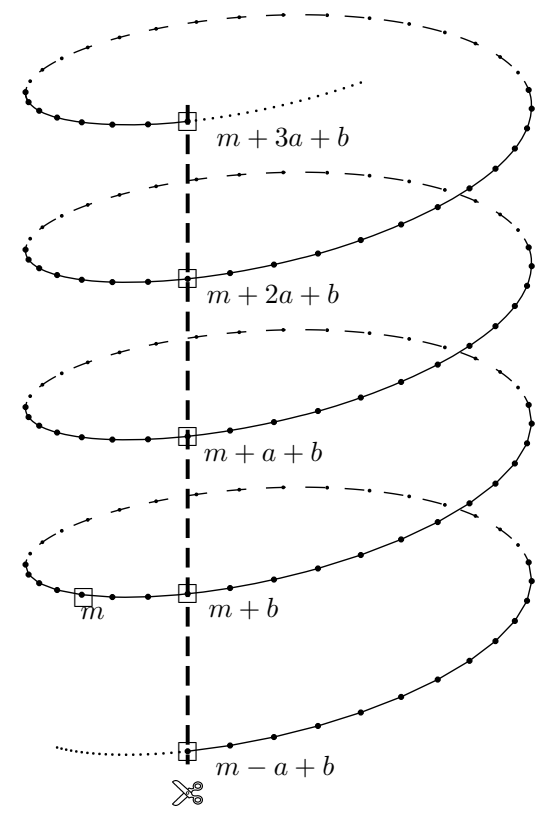

Figure 1. The integers on a helix

Since using the sketch of Figure 1 some of the integers would be hidden, so we will consider a planar projection of the cylinder instead. This projection is obtained by cutting the cylinder through a vertical line passing through a point previously chosen. Thus, every floor in this planar representation will contain exactly a fixed amount of elements. In Figure 1 we take this amount to be a positive integer $a$, and we cut along the vertical line passing through $m+b$ and $m+a+b$.

We shall use this drawings to depict the most relevant parts of the sets considered. For instance, if we want to highlight the elements of a numerical semigroup, we do not add any information by depicting the points below 0 and those above the conductor.

The parallelograms in Figure 2 highlight the elements of the semigroup $S=$ $\langle 9,13,15\rangle$, and the elements of $60-S$, respectively (choosing the size of the floor equal to 9$)$.
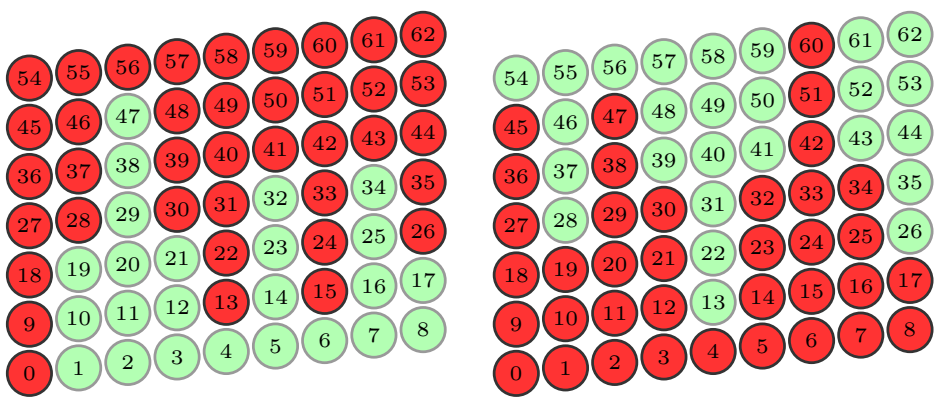

Figure 2. The semigroup $S=\langle 9,13,15\rangle$ and $60-S$, respectively 
Most times we are interested in finite sets of integers which are not smaller than a given integer $m$. In this case we prefer to draw all the points from $m$ to $m+a+b$ at the same level. See Figure 4 for an example. Its caption will soon become clear. For convenience, the columns are numbered. Having such a picture in mind, we can think of a partition of the set of integers greater than $m$ whose classes are the columns (the $i^{\text {th }}$ column of a set is the set of its elements congruent with $i$ modulo a).

\section{A GENERIC ALGORITHM}

We shall start the section by giving quite an efficient algorithm to compute the divisors of an element of a numerical semigroup. The aim is then to find an optimal configuration. Note that if $M$ is required to be an optimal configuration, we just have to control the cardinality of the difference $\mathrm{D}(M) \backslash \mathrm{D}(m)$, for all possible $m \in M$.

Among the optimal configurations there is an amenable set (Proposition 10). Thus, one can search for an optimal configuration among the amenable sets, which can be constructed using Algorithm 2, Due to the results in Subsection 3.3 (Corollary 14 to be more specific), one only needs to consider one amenable set for each shadow.

3.1. Divisors. Recall that given $x \in S$, we say that $\alpha \in S$ divides $x$ if $x-\alpha \in S$. We denote by $\mathrm{D}(x)$ the set of divisors of $x$, and $S_{x}=\{n \in S \mid n \leq x\}$.

Lemma 5. (1) $\mathrm{D}(x) \subseteq S_{x}$.

(2) $s \in \mathrm{D}(x)$ implies $\mathrm{D}(s) \subseteq \mathrm{D}(x)$.

(3) $\mathrm{D}(x)=S \cap(x-S)$.

(4) $\mathrm{D}(x)=S_{x} \cap\left(x-S_{x}\right)$.

Proof. The first assertion follows from the definition. If $s \in \mathrm{D}(x)$, then $x=s+y$ for some $y \in S$. For every $z \in \mathrm{D}(s), s=z+t$ for some $t \in s$, and thus $x=z+(t+y)$, which leads to $z \in \mathrm{D}(x)$. The rest of the statements are easy to prove.

The computation of the divisors of an element can be easily implemented (Algorithm 11) due to Lemma 5. Note also that, once we compute the elements of $S$ smaller than $x$ (which can easily be done if the conductor is known), the computation of the divisors is immediate.

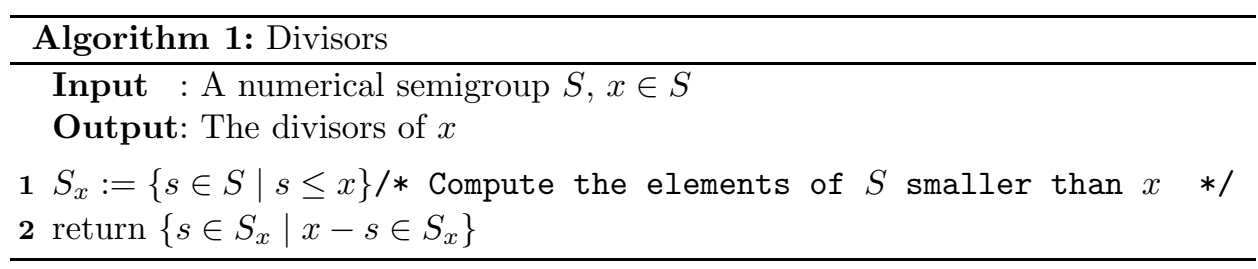

The highlighted elements in Figure 3 represent the divisors of $60 \in\langle 9,13,15\rangle$. They are obtained intersecting the highlighted elements of the pictures in Figure 2 , 


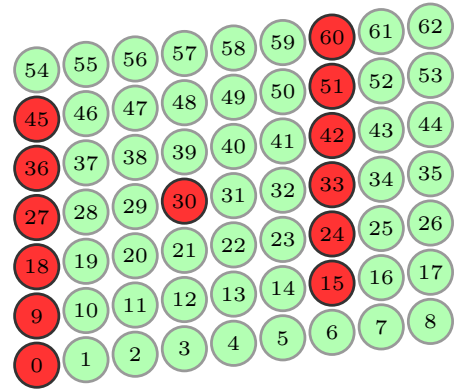

Figure 3. The divisors of 60 in the semigroup $S=\langle 9,13,15\rangle$

Another immediate consequence of Lemma 5, which has interest in concrete implementations, is the following corollary:

Corollary 6. If $c \leq x \leq y$, then $\mathrm{D}(y) \cap[x, \infty)=(y-S) \cap[x, \infty)$.

We remember that

$$
\begin{aligned}
\mathrm{D}\left(m_{1}, \ldots, m_{r}\right) & =\mathrm{D}\left(m_{1}\right) \cup \cdots \cup \mathrm{D}\left(m_{r}\right) \\
& =\left\{p \in S \mid m_{i}-p \in S \text { for some } i \in\{1, \ldots, r\}\right\} .
\end{aligned}
$$

The highlighted elements in Figure 4 are the elements of $\mathrm{D}(235,199,247,229)$ which are greater than 189 , when $S$ is the semigroup $\langle 19,20,21,22,23\rangle$.

Observe that $x-S$ contains all the integers not greater than $x-c$ and that the number of integers smaller than $x$ not belonging to $x-S$ is precisely the genus of $S$. As the number of non-negative integers not greater than $x$ is $x+1$, one gets immediately the well-known fact (see [11], [12] or [2]):

Proposition 7. If $x \geq 2 c-1$, then $\sharp \mathrm{D}(x)=\sharp S \cap(x-S)=x+1-2 g$.

\subsection{Amenable sets.}

Definition 8. Let $S$ be a numerical semigroup with conductor $c$, and let $M=$ $\left\{m_{1}<\cdots<m_{r}\right\} \subseteq S$. We say that $M$ is $m_{1}$-closed under division if

$$
\text { for all } i \in\{1, \ldots, r\}, \mathrm{D}\left(m_{i}\right) \cap\left[m_{1}, \infty\right) \subseteq M .
$$

If, in addition, $2 c-1 \leq m=m_{1}$, then we say that the set $M$ is $(S, m, r)$-amenable.

As a convention, the empty set is considered an $(S, m, 0)$-amenable set, for any $m$. When no confusion arises or only the concept is important, we say $(m, r)$-amenable set or simply amenable set.

Example 9. (1) Let $S=\langle 19,20,21,22,23\rangle$. Its conductor is $c=95$. Take $m=$ $2 c-1=189$. The set $M$ consisting of the highlighted elements in Figure 4 is an amenable subset of $S$.

(2) Let $S$ be a numerical semigroup with conductor $c$. Let $m \geq 2 c-1$, and let $r$ be a non-negative integer. Then the interval $[m, m+r-1] \cap \mathbb{N}$ is a $(S, m, r)$-amenable set. 


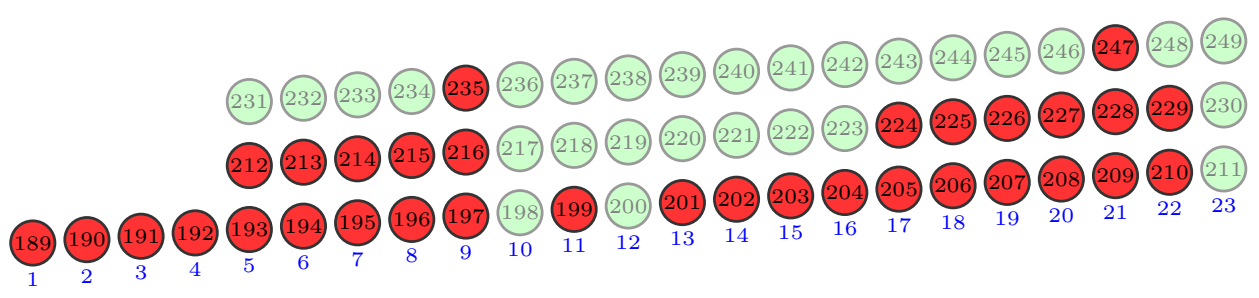

Figure 4. An amenable set

The importance of amenable sets comes from the following result, which states that among the optimal configurations of cardinality $r$ there is at least one $(S, m, r)$ amenable set.

Proposition 10. Let $S$ be a numerical semigroup with conductor $c$ and let $m \geq$ $2 c-1$. Let $r$ be a positive integer. Among the optimal configurations of cardinality $r$ there is one $(S, m, r)$-amenable set.

Proof. Let $M=\left\{m_{1}, \ldots, m_{r}\right\}$ be an optimal configuration. As $m \geq 2 c-1, \delta^{r}(m)$ is strictly increasing in $m$, and thus $m$ cannot be less than $m_{1}$, which implies that $m_{1}=m$.

If $M$ is not $m$-closed under division, we may assume that for some $i \in\{1, \ldots, r\}$ there exists $t \in S$ such that $m_{i}-t>m$ and $m_{i}-t \notin\left\{m_{1}, \ldots, m_{r}\right\}$. Clearly, $\mathrm{D}\left(m_{i}-\right.$ $t) \subset \mathrm{D}\left(m_{i}\right)$, and thus $\mathrm{D}\left(m_{1}, \ldots, m_{i-1}, m_{i}-t, m_{i+1}, \ldots, m_{r}\right) \subseteq \mathrm{D}\left(m_{1}, \ldots, m_{r}\right)$. In other words, we can change $m_{i}$ by $m_{i}-t$ and the number of divisors does not increase. Now we can repeat the process with the set obtained until we reach an $m$-closed under division set. Note that this must happen in a finite number of steps $\left(\mathbb{N}^{r}\right.$ has no infinite descending chains).

The definition of amenable set, which seems to be suitable for proofs, does not seem to help very much to do computations unless we can prove some consequences. The following one, showing that the distances between elements is somehow controlled, guarantees that the search of the amenable sets can be done in a bounded subset of $S$, and therefore amenable sets can be effectively computed. An algorithm will be presented (Algorithm 2).

Proposition 11. Let $S$ be a numerical semigroup with conductor $c$ and let $m \geq$ $2 c-1$. Let $M=\left\{m_{1}, \ldots, m_{r}\right\} \subseteq S$ be an $(S, m, r)$-amenable set and suppose that $S=\left\{0=\rho_{1}<\rho_{2}<\cdots\right\}$. Then

(a) $m_{i} \leq m+\rho_{i}$, for all $i \in\{1, \ldots, r\}$,

(b) $m_{i+1}-m_{i} \leq \rho_{2}$, for all $i \in\{1, \ldots, r-1\}$.

Proof. (a) Suppose that there exists $i_{0} \in\{1, \ldots, r\}$ such that $m_{i_{0}}-\rho_{i_{0}}>m$. Let $D=\left\{m_{i_{0}}-\rho_{j} \mid j \in\left\{1, \ldots, i_{0}\right\}\right\}$. All the elements of $D$ are bigger than $m$, that is, $D \subseteq(m, \infty)$. On the other hand, by using Lemma 5, $D \subseteq \mathrm{D}\left(m_{i_{0}}\right)$. Thus $D \subseteq \mathrm{D}\left(m_{i_{0}}\right) \cap(m, \infty) \varsubsetneqq\left\{m_{1}, \ldots, m_{i_{0}}\right\}$. The containment is strict since $m_{1}=m$. But this is absurd, since the two ends of the chain have the same cardinality.

(b) Note that $m_{i+1}-\rho_{2}$ is a divisor of $m_{i+1}$. This implies that, if $m_{i+1}-\rho_{2} \geq m$, then $m_{i+1}-\rho_{2} \in M$. As $m_{i+1}-\rho_{2}<m_{i+1}$ and there is no element in $M$ strictly between $m_{i}$ and $m_{i+1}, m_{i+1}-\rho_{2}$ must be not greater than $m_{i}$. 
For efficiency reasons, the following result is important. It shows that we do not have to consider all divisors.

Proposition 12. A subset $M=\left\{m=m_{1}, \ldots, m_{r}\right\}$ of a numerical semigroup $S$ is $(S, m, r)$-amenable if and only if

$$
\begin{aligned}
& \text { for all } i \in\{1, \ldots, r\} \text { and } g \text { minimal generator of } S \text {, } \\
& \text { if } m_{i}-g \geq m \text {, then } m_{i}-g \in\left\{m_{1}, \ldots, m_{r}\right\} \text {. }
\end{aligned}
$$

Proof. Let $m_{i} \in M$ and $u \in \mathrm{D}\left(m_{i}\right) \cap[m, \infty)$, with $u \neq m_{i}$. We shall prove that if (3) holds, then $u \in M$, thus concluding that $M$ is $(S, m, r)$-amenable. We can write $u=m_{i}-\gamma$, with $\gamma \in S \backslash\{0\}$. Assume as induction hypothesis that $m_{i}-\alpha \in \mathrm{D}\left(m_{i}\right) \cap[m, \infty)$ implies $m_{i}-\alpha \in M$, for all $\alpha$ less than $\gamma$. Let $g$ be a minimal generator that divides $\gamma$. As $\gamma-g<\gamma$, and $m_{i}-(\gamma-g)=m_{i}-\gamma+g \in$ $\mathrm{D}\left(m_{i}\right) \cap[m, \infty)$, we have, by hypothesis, that $m_{i}-\gamma+g \in M$. But then, by (3), $m_{i}-\gamma=\left(m_{i}-\gamma+g\right)-g \in M$.

Propositions 11 and 12 led to an algorithm to compute the set of $(S, m, r)$ amenable sets. Pseudo-code is presented in Algorithm 2 .

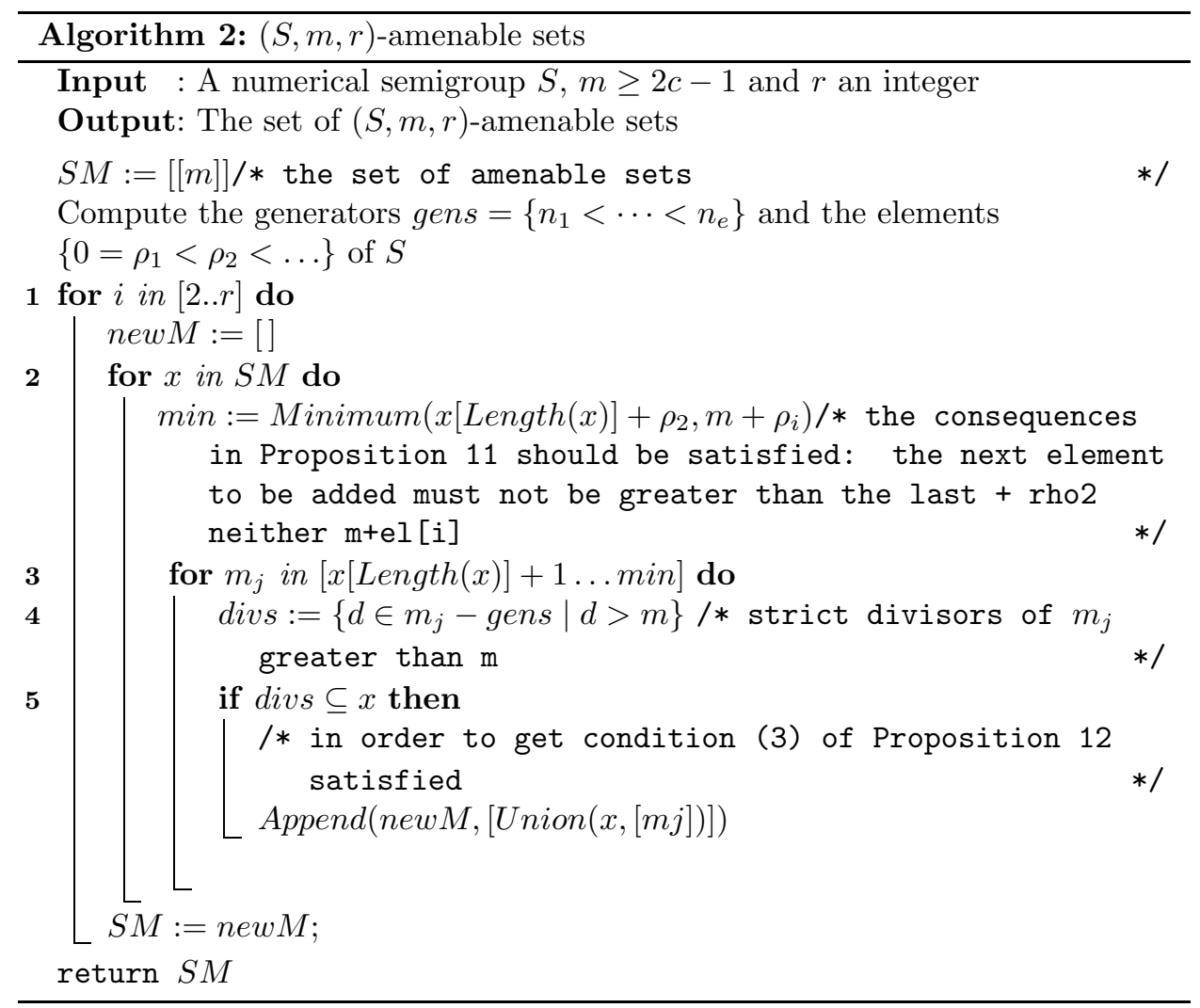

As we will see in the next subsection, we do not need all the amenable sets.

3.3. The ground. We continue considering $S$ a numerical semigroup minimally generated by $\left\{n_{1}<\cdots<n_{e}\right\}$ with conductor $c$. Let $m \geq 2 c-1$. The set $\left[m, m+n_{e}\right) \cap \mathbb{N}$ is called the $(S, m)$-ground, or simply ground. 
The intersection of an $(S, m, r)$-amenable set $M$ with the $(S, m)$-ground is called the shadow of $M$.

Note that the shadow of an amenable set is amenable.

Lemma 13. Let $S$ be a numerical semigroup minimally generated by $\left\{n_{1}<\cdots<\right.$ $\left.n_{e}\right\}$ with conductor $c$. Let $m \geq 2 c-1$ and let $M=\left\{m=m_{1}<\cdots<m_{r}\right\}$ be an amenable set. Let $L=M \cap\left[m, m+n_{e}\right)$ be the shadow of $M$. Then

$$
\mathrm{D}(M)=(M \backslash L) \cup \mathrm{D}(L),
$$

and furthermore $\sharp \mathrm{D}(M)=\sharp(M \backslash L)+\sharp \mathrm{D}(L)$.

Proof. The inclusion $(M \backslash L) \cup \mathrm{D}(L) \subseteq \mathrm{D}(M)$ is clear. For the other inclusion, let $x \in \mathrm{D}(M) \backslash(M \backslash L)=(\mathrm{D}(M) \backslash M) \cup L$. We want to prove that $x \in \mathrm{D}(L)$. Since $L \subseteq \mathrm{D}(L)$, we can assume that $x \in \mathrm{D}(M) \backslash M$. Then $x \in \mathrm{D}\left(m_{i}\right)$ for some $i \in\{1, \ldots, r\}$, and since $m_{i} \notin L, m_{i} \geq m+n_{e}$. As $m_{i}-x \in S \backslash\{0\}$, there exists $j \in\{1, \ldots, e\}$ such that $m_{i}-x-n_{j} \in S$. Hence $x \in \mathrm{D}\left(m_{i}-n_{j}\right)$. By hypothesis, $M$ is amenable and thus $m_{i}-n_{j} \in M$, since $m_{i}-n_{j} \in \mathrm{D}\left(m_{i}\right) \cap[m, \infty)$. If needed, we can repeat the process until $m_{i}-n_{j} \in L$, that is, $x \in \mathrm{D}(L)$.

The second assertion follows easily since the above union is disjoint.

As an easy but useful consequence, we get the following corollary.

Corollary 14. Let $M$ and $N$ be $(m, r)$-amenable sets with shadows $L_{M}$ and $L_{N}$, respectively. Then $L_{M} \subseteq L_{N} \Longrightarrow \sharp \mathrm{D}(M) \leq \sharp \mathrm{D}(N)$.

Proof. Suppose that $L_{N}$ is the disjoint union of $L_{M}$ and a set $K$ of cardinality $k$. Observe that, since both $M$ and $N$ have cardinality $r, \sharp\left(M \backslash L_{M}\right)=\sharp\left(N \backslash L_{N}\right)+k$.

As $\mathrm{D}\left(L_{N}\right)=\mathrm{D}\left(L_{M}\right) \cup \mathrm{D}(K) \supseteq \mathrm{D}\left(L_{M}\right) \cup K$, it follows that $\sharp \mathrm{D}\left(L_{N}\right) \geq \sharp \mathrm{D}\left(L_{M}\right)+k$, that is, $\sharp \mathrm{D}\left(L_{M}\right) \leq \sharp \mathrm{D}\left(L_{N}\right)-k$.

Thus, $\sharp \mathrm{D}(M)=\sharp\left(M \backslash L_{M}\right)+\sharp \mathrm{D}\left(L_{M}\right) \leq \sharp\left(N \backslash L_{N}\right)+k+\sharp \mathrm{D}\left(L_{N}\right)-k$.

Corollary 15. Let $S$ be a numerical semigroup minimally generated by $\left\{n_{1}<\right.$ $\left.\cdots<n_{e}\right\}$ with conductor $c$. Let $m \geq 2 c-1$ and let $M \subset[m, \infty)$ be an amenable set which is an optimal configuration of cardinality $r$. Let $L=M \cap\left[m, m+n_{e}\right)$ be the shadow of $M$. Then $\delta^{r}(m)=\sharp \mathrm{D}(L)+\sharp(M \backslash L)$.

Corollary 16. In particular, if there exists an optimal configuration $M$ of cardinality $r$ such that $\left[m, m+n_{e}\right) \cap \mathbb{N} \subseteq M$, then $[m, m+r-1+k] \cap \mathbb{N}$ is also an optimal configuration of cardinality $r+k$.

3.4. An algorithm to compute generalized Feng-Rao numbers. In the cases where computing divisors is "easy", finding optimal configurations is as difficult as computing generalized Feng-Rao numbers. This problem is referred to as "hard" in the literature, even from the computational point of view.

Algorithm 3 can be used to compute generalized Feng-Rao numbers of any numerical semigroup. Note that its efficiency depends on the number of amenable sets. Due to Corollary 14, it can be sharpened, since we only need to consider one amenable set for each possible shadow. 


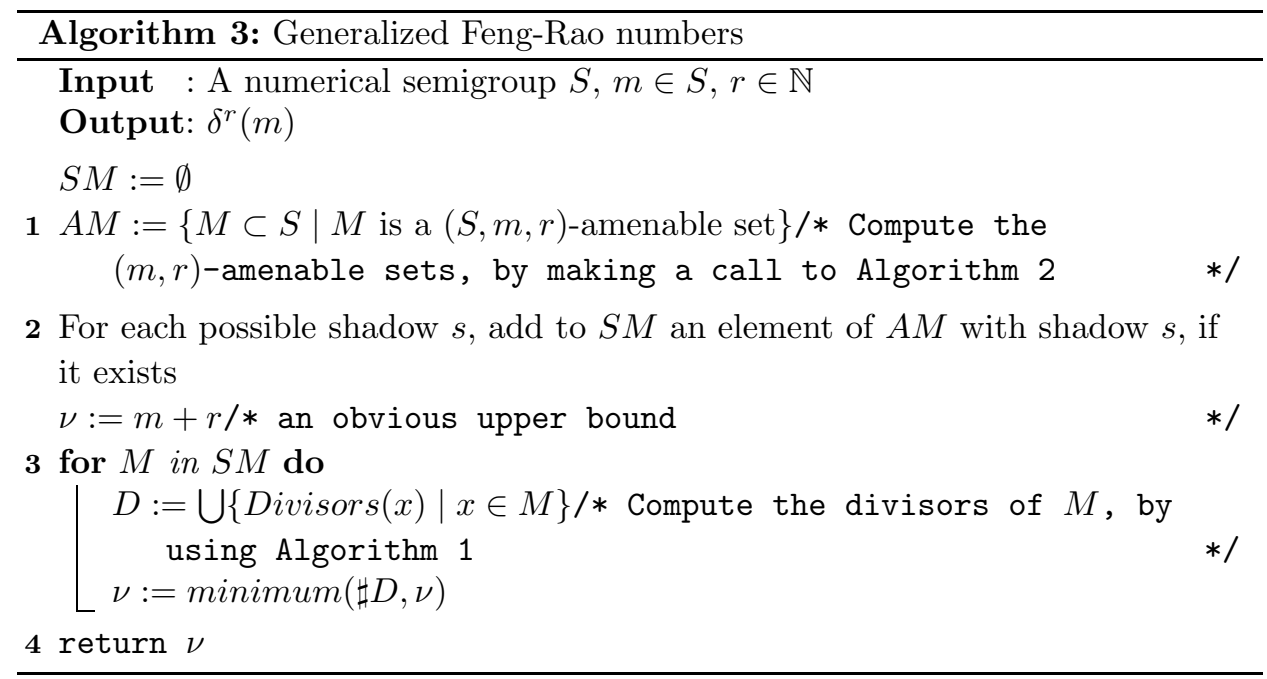

This algorithm (even preliminary versions of it) has been extensively used by the authors to perform computations which gave the intuition that ultimately led to the main results of this paper.

\section{Numerical SEMigroups Generated By intervals}

From now on we assume that $S=\langle a, \ldots, a+b\rangle$ with $a$ and $b$ positive integers, and $b<a$.

4.1. Some counting lemmas. As we have seen above, it is crucial to know the number of divisors of subsets of the ground (this is obtained in Remark 22). In this section we prove some technical lemmas on counting the divisors of elements, and then apply them for elements in the ground. The main result (Proposition 28) shows that the minimum is obtained when the elements form an interval starting in $m$.

The membership problem for semigroups generated by intervals is trivial as the following known result (and with many different formulations) shows.

Lemma 17 ([4, Lemma 10, for $d=1])$. Let $k$ and $r$ be integers such that $0 \leq r \leq$ $a-1$. Then $k a+r \in S$ if and only if $r \leq k b$.

Lemma 18. Let $m \geq 2 c-1$. Let $q$ be a non-negative integer and $j \in\{0, \ldots, a-1\}$.

$$
\begin{aligned}
\mathrm{D}(m, m+ & q a+j)=\mathrm{D}(m) \\
& \cup\left\{m-(k a+r) \mid 0 \leq r \leq a-j-1, \frac{r+j}{b}-q \leq k<\frac{r}{b}\right\} \\
& \cup\left\{m-(k a+r) \mid a-j \leq r \leq a-1, \frac{r+j-(a+b)}{b}-q \leq k<\frac{r}{b}\right\},
\end{aligned}
$$

and this union is disjoint.

Proof. We describe the set $\mathrm{D}(m+q a+j) \backslash \mathrm{D}(m)$. Let $x$ be an integer such that $m+q a+j-x \in S$ and $m-x \notin S$. In particular, as $m \geq 2 c-1, m-x \notin S$ implies that $m-x<c$, and thus $c-1 \leq m-c<x$, which leads to $x \in S$. Thus $x \in \mathrm{D}(m+q a+j) \backslash \mathrm{D}(m)$. Set $n=m-x$, and let $k$ and $r$ be integers such 
that $n=k a+r(x=m-(k a+r))$. Then $n=k a+r \notin S$ and $n+q a+j=$ $(q+k) a+(j+r) \in S$. In view of Lemma 17 this implies that $k b<r<a$ and

- if $r+j \leq a-1$, then $0 \leq r+j \leq(q+k) b$,

- if $r+j \geq a$, by writing $(q+k) a+(j+r)=(q+k+1) a+(j+r-a)$, we obtain $0 \leq r+j-a \leq(q+k+1) b$.

Figure[5] shows the divisors of $\mathrm{D}(m, m+\lambda)$ with $m=2 c-1=35, m+\lambda \in\{42,59\}$, and $S=\langle 9,10,11,12,13\rangle$.

divisors of $m$ in the ground

divisors of $m$ divisors of $m+\lambda$ in the ground

divisors of $m$ and $m+\lambda$

Other elements in the ground

Other elements
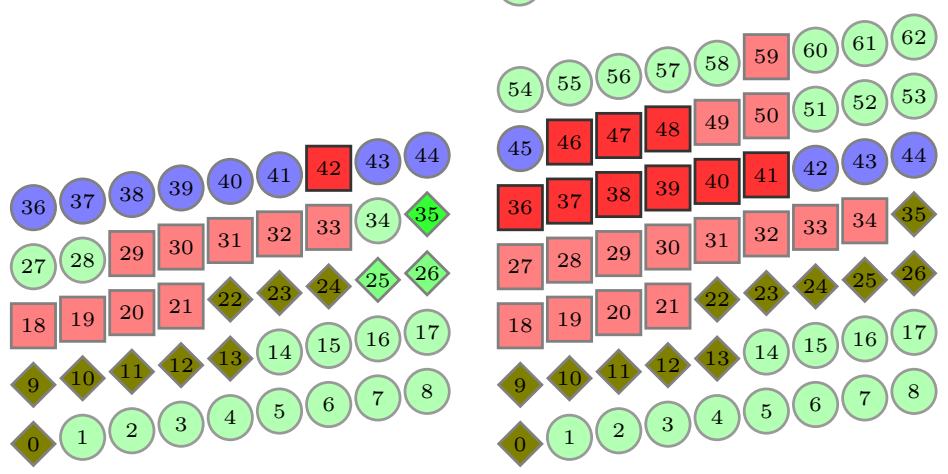

Figure 5. $\mathrm{D}(m, m+\lambda)$

Remark 19. For the particular case $q=0$ and $0<j<a$, we get

$\mathrm{D}(m, m+j)=\mathrm{D}(m) \cup\{m-(k a+r) \mid a-j \leq r \leq a-1,0<r-k b \leq(a+b)-j\}$.

For $q=1$ and $j=0$,

$$
\mathrm{D}(m, m+a)=\mathrm{D}(m) \cup\{m-(k a+r) \mid 0 \leq r \leq a-1,0<r-k b \leq b\},
$$

which is the same as above by taking $j=a$.

For the case $q=1$, we get

$$
\begin{aligned}
\mathrm{D}(m, m+a+j) & =\mathrm{D}(m) \\
& \cup\{m-(k a+r) \mid 0 \leq r \leq a-j-1,0<r-k b \leq b-j\} \\
& \cup\{m-(k a+r) \mid a-j \leq r \leq a-1,0<r-k b \leq(a+b)+b-j\} .
\end{aligned}
$$

This describes all elements $\mathrm{D}(m, m+\ell)$ with $\ell \in\{1, \ldots, a+b-1\}$ (i.e., $m+\ell$ in the ground). 
Lemma 20. Let $0=i_{0}<i_{1}<\cdots<i_{t}<i_{t+1}<a+b$ be such that $\{m, m+$ $\left.i_{1}, \ldots, m+i_{t+1}\right\}$ is amenable. Then

$$
\begin{aligned}
& \mathrm{D}\left(m, m+i_{1}, \ldots, m+i_{t+1}\right)=\mathrm{D}\left(m, m+i_{1}, \ldots, m+i_{t}\right) \\
& \quad \cup\left\{m-(k a+r) \mid a-i_{t+1} \leq r \leq a-1-i_{t}, 0<r-k b \leq(a+b)-i_{t+1}\right\} .
\end{aligned}
$$

Proof. Assume first that $i_{t+1} \leq a$. Note that $\mathrm{D}\left(m+i_{t+1}\right) \backslash\left(\mathrm{D}\left(m, m+i_{1}, \ldots, m+\right.\right.$ $\left.\left.i_{t}\right)\right)=\bigcap_{j=0}^{t} \mathrm{D}\left(m+i_{t+1}\right) \backslash \mathrm{D}\left(m+i_{j}\right)$, and this equals

$$
\bigcap_{j=0}^{t}\left\{m+i_{j}-\left(k a+r^{\prime}\right) \mid a-\left(i_{t+1}-i_{j}\right) \leq r^{\prime} \leq a-1,0<r^{\prime}-k b \leq(a+b)-\left(i_{t+1}-i_{j}\right)\right\}
$$

(Remark 19). If we make the change of variables $r=r^{\prime}-i_{j}$ for each $j$, we obtain

$$
\bigcap_{j=0}^{t}\left\{m-(k a+r) \mid a-i_{t+1} \leq r \leq a-1-i_{j},-i_{j}<r-k b \leq(a+b)-i_{t+1}\right\} .
$$

Intersecting means choosing the least intervals for $r$ and $r-k b$, and we get the desired result.

Now assume that $a<i_{t+1}<a+b$. By hypothesis there exists $s$ such that $i_{t+1}-i_{s}<a$ and $i_{t+1}-i_{s-1} \geq a$ (by amenability). For $i_{t+1}-i_{j} \geq a$, write $i_{t+1}-i_{j}=a+h_{j}$. Hence $\bigcap_{j=0}^{t} \mathrm{D}\left(m+i_{t+1}\right) \backslash \mathrm{D}\left(m+i_{j}\right)$ equals

$$
\begin{aligned}
& \bigcap_{j=s}^{t}\left\{m+i_{j}-\left(k a+r^{\prime}\right) \mid a-\left(i_{t+1}-i_{j}\right) \leq r^{\prime} \leq a-1,0<r^{\prime}-k b \leq(a+b)-\left(i_{t+1}-i_{j}\right)\right\} \\
& \bigcap\left(\bigcap _ { j = 0 } ^ { s - 1 } \left(\left\{m+i_{j}-\left(k a+r^{\prime}\right) \mid 0 \leq r^{\prime} \leq a-\left(i_{t+1}-i_{j}-a\right)-1,0<r^{\prime}-k b\right.\right.\right. \\
& \left.\leq b-\left(i_{t+1}-i_{j}-a\right)\right\} \cup\left\{m+i_{j}-\left(k a+r^{\prime}\right) \mid a-\left(i_{t+1}-i_{j}-a\right)\right. \\
& \left.\left.\left.\leq r^{\prime} \leq a-1,0<r^{\prime}-k b \leq(a+b)+b-\left(i_{t+1}-i_{j}-a\right)\right\}\right)\right) .
\end{aligned}
$$

If we perform again the change of variables $r=r^{\prime}-i_{j}$, we obtain that $C=$ $\bigcap_{j=s}^{t}\left\{m+i_{j}-\left(k a+r^{\prime}\right) \mid a-\left(i_{t+1}-i_{j}\right) \leq r^{\prime} \leq a-1,0<r^{\prime}-k b \leq(a+b)-\left(i_{t+1}-i_{j}\right)\right\}=$ $\left\{m-(k a+r) \mid a-i_{t+1} \leq r \leq a-i_{t}-1,-i_{s}<r-k b \leq(a+b)-i_{t+1}\right\}$. Analogously, for every $j \in\{0, \ldots, s-1\}$,

$$
\begin{gathered}
\left\{m+i_{j}-\left(k a+r^{\prime}\right) \mid 0 \leq r^{\prime} \leq a-\left(i_{t+1}-i_{j}-a\right)-1,0<r^{\prime}-k b \leq b-\left(i_{t+1}-i_{j}-a\right)\right\} \\
\cup\left\{m+i_{j}-\left(k a+r^{\prime}\right) \mid a-\left(i_{t+1}-i_{j}-a\right) \leq r^{\prime} \leq a-1,0<r^{\prime}-k b\right. \\
\left.\leq(a+b)+b-\left(i_{t+1}-i_{j}-a\right)\right\}
\end{gathered}
$$

equals

$$
\begin{aligned}
& \left\{m-(k a+r) \mid-i_{j} \leq r \leq 2 a-i_{t+1}-1,-i_{j}<r-k b \leq a+b-i_{t+1}\right\} \\
& \cup\left\{m-(k a+r) \mid 2 a-i_{t+1} \leq r \leq a-i_{j}-1,-i_{j}<r-k b \leq 2(a+b)-i_{t+1}\right\} .
\end{aligned}
$$

Observe that $a-i_{t}-1 \leq 2 a-i_{t+1}-1$ if and only if $i_{t+1}-i_{t} \leq a$, which is the case since we are using an amenable set. Hence $C$ does not cut the second set in the above union, and the whole intersection is as in the case $i_{t+1}<a$. 
Corollary 21. Let $0=i_{0}<i_{1}<\cdots<i_{t}<i_{t+1}<a+b$. Then

$$
\begin{aligned}
\sharp \mathrm{D}\left(m, m+i_{1}, \ldots, m+i_{t+1}\right)= & \sharp \mathrm{D}\left(m, m+i_{1}, \ldots, m+i_{t}\right) \\
& +\sum_{j=i_{t}+1}^{i_{t+1}}\left\lceil\frac{a+b-j}{b}\right\rceil-\left\lceil\frac{i_{t+1}-j}{b}\right\rceil .
\end{aligned}
$$

Proof. We compute the cardinality of $\left\{m-(k a+r) \mid a-i_{t+1} \leq r \leq a-1-\right.$ $\left.i_{t}, 0<r-k b \leq(a+b)-i_{t+1}\right\}$. Note that $m-(k a+r)=m-\left(k^{\prime} a+r^{\prime}\right)$ with $0 \leq r, r^{\prime}<a$ implies that $k=k^{\prime}$ and $r=r^{\prime}$. Thus we must calculate $\sharp\left\{(k, r) \mid a-i_{t+1} \leq r \leq a-1-i_{t}, 0<r-k b \leq(a+b)-i_{t+1}\right\}$, which equals $\sharp\left\{(k, r) \mid i_{t}+1 \leq a-r \leq i_{t+1}, \frac{i_{t+1}+r-(a+b)}{b} \leq k<\frac{r}{b}\right\}$. By taking $j=a-r$, we get

$$
\begin{aligned}
\sum_{j=i_{t}+1}^{i_{t+1}} \sharp\left\{k \mid \frac{i_{t+1}-j}{b}-1 \leq k<\frac{a-j}{b}\right\} \\
\quad=\sum_{j=i_{t}+1}^{i_{t+1}}\left(\left(\left\lceil\frac{a-j}{b}\right\rceil-1\right)-\left(\left\lceil\frac{i_{t+1}-j}{b}\right\rceil-1\right)+1\right),
\end{aligned}
$$

and the proof follows easily.

Remark 22. Recall that

$$
\begin{aligned}
\sharp \mathrm{D}\left(m, m+i_{1}, \ldots, m+i_{t}\right)= & \sharp \mathrm{D}\left(m, m+i_{1}, \ldots, m+i_{t-1}\right) \\
& +\sum_{j=i_{t-1}+1}^{i_{t}}\left\lceil\frac{a+b-j}{b}\right\rceil-\left\lceil\frac{i_{t}-j}{b}\right\rceil,
\end{aligned}
$$

and by applying this process several times we obtain

$$
\begin{aligned}
\sharp \mathrm{D}\left(m, m+i_{1}, \ldots, m+i_{t}\right) & =\sharp \mathrm{D}(m)+\sum_{k=1}^{t} \sum_{j=i_{k-1}+1}^{i_{k}}\left\lceil\frac{a+b-j}{b}\right\rceil-\left\lceil\frac{i_{k}-j}{b}\right\rceil \\
& =\sharp \mathrm{D}(m)+\sum_{j=1}^{i_{t}}\left\lceil\frac{a+b-j}{b}\right\rceil-\sum_{k=1}^{t} \sum_{j=i_{k-1}+1}^{i_{k}}\left\lceil\frac{i_{k}-j}{b}\right\rceil .
\end{aligned}
$$

Thus

$\sharp \mathrm{D}\left(m, m+i_{1}, \ldots, m+i_{t}\right)=\sharp \mathrm{D}(m)+\sum_{j=1}^{i_{t}}\left\lceil\frac{a+b-j}{b}\right\rceil-\sum_{k=1}^{t} \sum_{j=1}^{i_{k}-i_{k-1}}\left\lceil\frac{\left(i_{k}-i_{k-1}\right)-j}{b}\right\rceil$.

If we write $d_{k}=i_{k}-i_{k-1}$, this rewrites as

$$
\sharp \mathrm{D}\left(m, m+i_{1}, \ldots, m+i_{t}\right)=\sharp \mathrm{D}(m)+\sum_{j=1}^{i_{t}}\left\lceil\frac{a+b-j}{b}\right\rceil-\sum_{k=1}^{t} \sum_{j=1}^{d_{k}}\left\lceil\frac{d_{k}-j}{b}\right\rceil .
$$

Hence the value of $\sharp \mathrm{D}\left(m, m+i_{1}, \ldots, m+i_{t}\right)$ depends on $d_{1}, \ldots, d_{t}$, subject to $\sum_{k=1}^{t} d_{k}=i_{t}$.

Corollary 23. Let $t \in\{1, \ldots, a+b-1\}$. Then

$$
\sharp \mathrm{D}(m, m+1, \ldots, m+t)=\sharp \mathrm{D}(m)+\sum_{j=1}^{t}\left\lceil\frac{a+b-j}{b}\right\rceil .
$$


As a consequence of this, when the shadow of a configuration is an interval containing $m$, then we can compute the number of divisors of its elements.

Corollary 24. Let $m$ be an integer greater than or equal to $2 c-1$. Let $m=m_{1}<$ $m_{2}<\cdots<m_{t}$ be integers such that $\left\{m_{1}, m_{2}, \ldots, m_{t}\right\}$ is amenable. Assume that $l=\sharp\left\{m_{1}, \ldots, m_{t}\right\} \cap[m, m+a+b)=\{m, m+1, \ldots, m+l-1\}$. Then

$$
\sharp \mathrm{D}\left(m_{1}, \ldots, m_{t}\right)=m-2 g+t+\sum_{j=1}^{l-1}\left\lceil\frac{a-j}{b}\right\rceil .
$$

Proof. This is a direct consequence of Proposition 7, Lemma 13, and Corollary 23 .

Indeed, we will show that among these configurations there is an optimal one. To do this, we first prove that the best shadows are those of the form $\{m, m+$ $1, \ldots, m+l-1\}$, and later (in the next section) we will have to compute the smallest possible value of $l$.

Let us see how to compute sums of the form $\sum_{j=1}^{t}\left\lceil\frac{a-j}{b}\right\rceil$.

Lemma 25. Let $x$ and $y$ be postive integers. Assume that $y=c b+r$ with $c$ an integer and $0 \leq r<b$, and that $k$ is an integer such that $k b \leq x-r<(k+1) b$. Then

(1) if $r \neq 0, \sum_{j=1}^{x}\left\lceil\frac{y-j}{b}\right\rceil=(c+1)(r-1)+b \sum_{i=0}^{k-1}(c-i)+(x-(k b+r)+1)(c-k)=$ $x(c-k)+(k+1)(r-1)+b \frac{k(k+1)}{2}$,

(2) if $r=0, \sum_{j=1}^{x}\left\lceil\frac{y-j}{b}\right\rceil=-c+b \sum_{i=0}^{k-1}(c-i)+(x-k b+1)(c-k)=$ $(x+1)(c-k)+b \frac{k(k+1)}{2}-c$.

Proof. Observe that

$\sum_{j=1}^{x}\left\lceil\frac{y-j}{b}\right\rceil=\sum_{j=1}^{r-1}\left\lceil\frac{y-j}{b}\right\rceil+\sum_{j=r}^{r+b-1}\left\lceil\frac{y-j}{b}\right\rceil+\cdots+\sum_{j=(k-1) b+r}^{k b+r-1}\left\lceil\frac{y-j}{b}\right\rceil+\sum_{j=k b+r}^{x}\left\lceil\frac{y-j}{b}\right\rceil$,

and

$$
\sum_{j=r+l b}^{(l+1) b+r-1}\left\lceil\frac{y-j}{b}\right\rceil=\sum_{j=0}^{b-1}\left\lceil\frac{y-(r+l b)-j}{b}\right\rceil=\sum_{j=0}^{b-1}\left\lceil\frac{(c-l) b-j}{b}\right\rceil=b(c-l) .
$$

In the same way, the first and last summand are computed. If $r=0$, the first summand does not appear, and the second sum starts on 0 , and so we have to decrease the total amount by $\left\lceil\frac{c b}{b}\right\rceil=c$.

Actually, as we see next it suffices to consider the following type of sums.

Remark 26. For the case $x=y$, we get $k=c$ and

(1) if $r \neq 0, \sum_{j=1}^{x}\left\lceil\frac{x-j}{b}\right\rceil=(c+1)(r-1)+b \frac{c(c+1)}{2}=\frac{c+1}{2}(x+r)-c-1$,

(2) if $r=0, \sum_{j=1}^{x}\left\lceil\frac{x-j}{b}\right\rceil=\frac{c+1}{2} x-c$.

Observe also that $\sum_{j=1}^{x}\left\lceil\frac{x-j}{b}\right\rceil=\sum_{j=1}^{x-1}\left\lceil\frac{j}{b}\right\rceil$. 
The following trick will allow us to prove that the best possible shadows are those that are intervals starting in $m$.

Remark 27. Let $d_{k}=i_{k}-i_{k-1}, i \in\{1, \ldots, t\}$. Then $\sum_{k=1}^{t} d_{k}=i_{t}$. If we replace $\left\{d_{1}, d_{2}\right\}$ with $\left\{1, d_{1}+d_{2}-1\right\}$, the total sum of the $d_{k}$ 's remains the same (we are thus assuming that both $d_{1}$ and $d_{2}$ are greater than one). Let us see what happens to the following:

$$
\sum_{k=1}^{t} \sum_{j=1}^{i_{k}-i_{k-1}}\left\lceil\frac{\left(i_{k}-i_{k-1}\right)-j}{b}\right\rceil=\sum_{k=1}^{t} \sum_{j=1}^{d_{k}}\left\lceil\frac{d_{k}-j}{b}\right\rceil .
$$

Write $d_{k}=c_{k} b+r_{k}$, with $c_{k}$ and $r_{k}$ integers such that $0 \leq r_{k}<b$. Set $s_{k}=$ $\sum_{j=1}^{d_{k}}\left\lceil\frac{d_{k}-j}{b}\right\rceil$. Then $s_{k}=\frac{c_{k}+1}{2}\left(d_{k}+r_{k}\right)-1-c_{k}$, if $r_{k} \neq 0$, and $s_{k}=\frac{c_{k}+1}{2} d_{k}-c_{k}$, otherwise. Let $c$ and $r$ be the quotient and remainder of the division of $d_{1}+d_{2}-1$ by $b$. Let $\Delta=\sum_{j=1}^{d_{1}+d_{2}-1}\left[\frac{d_{1}+d_{2}-1-j}{b}\right]-s_{1}-s_{2}$. If $b=1$, then $r_{1}=r_{2}=r=0$, $c_{i}=d_{i}, c=d_{1}+d_{2}-1$, and $\Delta=d_{1} d_{2}$, which is a non-negative integer. For $b>1$ we distinguish three cases depending on the value of $r_{1}+r_{2}$.

- If $r_{1}+r_{2}=0$ (this means $r_{1}=r_{2}=0$ ), then $d_{1}=c_{1} b, d_{2}=c_{2} b, c=$ $c_{1}+c_{2}-1$, and $r=b-1$. Then $\Delta=b c_{1} c_{2}-\left(c_{1}+c_{2}\right)$. Since we are assuming that $b \geq 2$, and $c_{1}$ and $c_{2}$ are positive integers, this amount is non-negative.

- If $0<r_{1}+r_{2} \leq b$, then $c=c_{1}+c_{2}$ and $r=r_{1}+r_{2}-1$. Thus, if $r r_{1} r_{2} \neq 0$, $\Delta=b c_{1} c_{2}+c_{1}\left(r_{2}-1\right)+c_{2}\left(r_{1}-1\right)$, which is greater than or equal to zero. For $r=0$, either $r_{1}=0$ (and $r_{2}=1$ ) or $r_{2}=0$ (and $r_{1}=1$ ). Assume without loss of generality that $r_{1}=0$. We obtain $\Delta=\left(b c_{1}-1\right) c_{2}$, which is again non-negative.

- Finally, if $r_{1}+r_{2} \geq b+1$, then $c=c_{1}+c_{2}+1$ and $r=r_{1}+r_{2}-b-1$. In this setting $r_{1} \neq 0 \neq r_{2}$. If $r \neq 0$, then $\Delta=b c_{1} c_{2}+c_{1}\left(r_{2}-1\right)+c_{2}\left(r_{1}-1\right)+r_{1}+$ $r_{2}-(b+2)$, which is non-negative since $r_{1}+r_{2} \geq b+2$. For $r_{1}+r_{2}=b+1$ $(r=0)$, we obtain a non-negative $\Delta=b c_{1} c_{2}+c_{1}\left(r_{2}-1\right)+c_{2}\left(r_{1}-1\right)$.

With all of this in mind, we are able to prove the main result of this section, that is, if the elements in the ground form an interval containing $m$, then we get the least possible number of divisors.

Proposition 28. Let $0=i_{0}<i_{1}<\cdots<i_{t}<a+b$. Then

$$
\sharp \mathrm{D}\left(m, m+i_{1}, \ldots, m+i_{t}\right) \geq \sharp \mathrm{D}(m, m+1, \ldots, m+t) .
$$

Proof. Let $d_{k}=i_{k}-i_{k-1}$ for $k \in\{1, \ldots, t\}\left(i_{0}=0\right)$. We know that (see Remark 22)

$$
\sharp \mathrm{D}\left(m, m+i_{1}, \ldots, m+i_{t}\right)=\sharp \mathrm{D}(m)+\sum_{j=1}^{i_{t}}\left\lceil\frac{a+b-j}{b}\right\rceil-\sum_{k=1}^{t} \sum_{j=1}^{d_{k}}\left\lceil\frac{d_{k}-j}{b}\right\rceil .
$$

By applying the above remark several times, we obtain that $\sharp \mathrm{D}\left(m, m+i_{1}, \ldots, m+\right.$ $\left.i_{t}\right) \geq \sharp \mathrm{D}\left(m, m+i_{1}^{\prime}, m+i_{2}^{\prime}, \ldots, m+i_{t}^{\prime}\right)$, with $i_{t}=i_{t}^{\prime}$ and $d_{k}^{\prime}=i_{k}^{\prime}-i_{k-1}^{\prime}=1$ for $k \in\{2, \ldots, t\}$. By using again the above expression, but now for $d_{k}^{\prime}$ instead of $d_{k}$, we get

$$
\sharp \mathrm{D}\left(m, m+i_{1}^{\prime}, \ldots, m+i_{t}^{\prime}\right)=\sharp \mathrm{D}(m)+\sum_{j=1}^{i_{t}}\left\lceil\frac{a+b-j}{b}\right\rceil-\sum_{j=1}^{i_{1}^{\prime}}\left\lceil\frac{i_{1}^{\prime}-j}{b}\right\rceil .
$$


Hence, by Corollary 23, in order to prove the inequality of the statement, it suffices to show that

$$
\sum_{j=1}^{i_{t}}\left\lceil\frac{a+b-j}{b}\right\rceil-\sum_{j=1}^{i_{1}^{\prime}}\left\lceil\frac{i_{1}^{\prime}-j}{b}\right\rceil \geq \sum_{j=1}^{t}\left\lceil\frac{a+b-j}{b}\right\rceil,
$$

or equivalently,

$$
\sum_{j=t+1}^{i_{t}}\left\lceil\frac{a+b-j}{b}\right\rceil-\sum_{j=1}^{i_{1}^{\prime}}\left\lceil\frac{i_{1}^{\prime}-j}{b}\right\rceil \geq 0
$$

Now, if we take into account that $\sum_{j=t+1}^{i_{t}}\left\lceil\frac{a+b-j}{b}\right\rceil=\sum_{j=1}^{i_{t}-t}\left\lceil\frac{a+b-t-j}{b}\right\rceil$, that $\sum_{j=1}^{i_{1}^{\prime}}\left\lceil\frac{i_{1}^{\prime}-j}{b}\right\rceil=\sum_{j=1}^{i_{1}^{\prime}-1}\left\lceil\frac{i_{1}^{\prime}-j}{b}\right\rceil$, and that $i_{1}^{\prime}+(t-1)=i_{t}^{\prime}=i_{t}$, we get $\sum_{j=t+1}^{i_{t}}\left\lceil\frac{a+b-j}{b}\right\rceil$ $=\sum_{j=1}^{i_{1}^{\prime}-1}\left\lceil\frac{a+b-t-j}{b}\right\rceil$. Since $a+b-t \geq i_{t}+1-t=i_{1}^{\prime}$, we obtain the desired inequality.

4.2. Ordered amenable sets. As we have seen above, the minimum number of divisors of elements in the ground is reached when these elements form an interval starting in $m$. In this section we study configurations fulfilling this condition.

Let $M$ be a configuration and let

$$
j_{0}=\max \{j \in\{0, \ldots, a-1\} \mid x-(m+b)=q a+j, \text { for some } q \in \mathbb{Z} \text { and } x \in M\} .
$$

Let us call wagon of $M$ the set $\left\{x \in M \mid x-(m+b)=q a+j_{0}\right.$, for some integer $\left.q\right\}$.

An element $P$ of a configuration $M$ is said to be the pivot of $M$ if either $(P<$ $m+b$ and $P$ is the maximum of $M$ ) or, $P$ is the maximum of the wagon.

Note that the wagon and the pivot element of a configuration can be determined in an algorithmic way.

Checking Figure 6 may be useful $(a=19, b=4)$. The wagon is column 22 and the pivot is 305 .

The wagon consists of the rightmost elements of $M$. The highest of these is the pivot element. Note that the index of the column containing the wagon is $b+j_{0}$.

Definition 29. An $(S, m, r)$-amenable set $M$ with pivot element $P$ is said to be ordered amenable if its shadow is of the form $\{m, m+1, \ldots, m+t\}$, for some integer $t, 0 \leq t<a+b-1$, and the only element that can possibly be added to obtain an $(S, m, r+1)$-amenable set without increasing the shadow is $P+\rho_{2}$. 


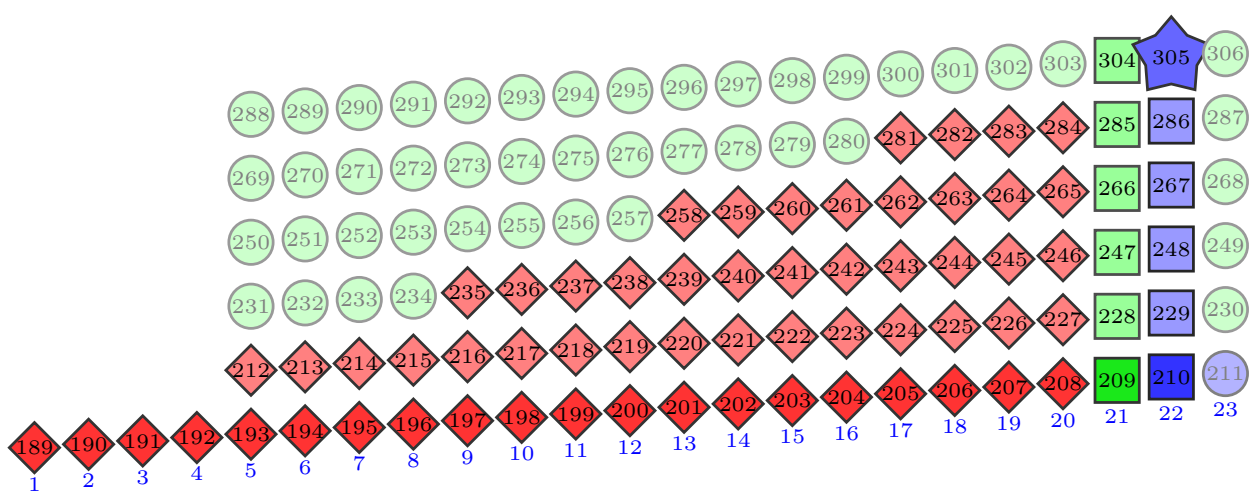

FiguRE 6. The biggest ordered amenable.

We now show how to construct $(S, m, r)$-amenable sets.

Remark 30. In view of Lemma 18, for every positive integer $q$ with $q b<a$,

$\mathrm{D}(m+q a+q b)=\mathrm{D}(m) \cup\left\{m-(k a+r) \mid a-q b \leq r \leq a-1, \frac{r-(a+b)}{b} \leq k<\frac{r}{b}\right\}$.

By performing a change of variables (change $a-r$ to $r$ and $-k-1$ to $k$ ), we obtain that

$$
\mathrm{D}(m+q a+q b)=\mathrm{D}(m) \cup\left\{m+k a+r \mid 1 \leq r \leq q b,-\frac{a-r}{b}-1<k \leq \frac{r}{b}\right\} .
$$

Hence

$$
\mathrm{D}(m+q a+q b) \cap[m, \infty)=\{m\} \cup\left\{m+k a+r \mid 1 \leq r \leq q b, 0 \leq k \leq \frac{r}{b}\right\}
$$

and

$$
\mathrm{D}(m+q a+q b) \cap[m, m+a+b)=\{m, m+1, \ldots, m+q b\}
$$

(observe that for $k$ to be one, $r$ must be at least $b$, and in this case we obtain $m+a+b$ which is not in $[m, m+a+b))$.

Moreover,

$$
\sharp \mathrm{D}(m+q a+q b) \cap[m, \infty)=1+q+\frac{b}{2} q(q+1),
$$

since the cardinality of the set $\left\{m+k a+r \mid 1 \leq r \leq q b, 0 \leq k \leq \frac{r}{b}\right\}$ is $\sum_{r=1}^{q b}\left(\left\lfloor\frac{r}{b}\right\rfloor+1\right)$, which can be rewritten as $q b+\sum_{i=0}^{q-1} \sum_{j=0}^{b-1}\left\lfloor\frac{i b+j}{b}\right\rfloor+\left\lfloor\frac{q b}{b}\right\rfloor$, and this equals $q b+q+$ $\sum_{i=0}^{q-1} b i=q b+q+b \frac{q(q-1)}{2}=q+\frac{b}{2} q(q+1)$. Now by adding the cardinality of $\{m\}$, we obtain the desired equality.

Clearly the sets $\mathrm{D}(m+\lambda) \cap[m, \infty)$ are amenable sets. The following lemma shows that some of these are indeed ordered amenable sets. The problem is that their cardinalities do not cover all possible $r$ 's.

Lemma 31. Let $q$ be a positive integer such that $q b<a$. Then $\mathrm{D}(m+q a+q b) \cap$ $[m, \infty)$ is an ordered amenable set.

Proof. We already know that its shadow is an interval containing $m$ (the condition $q b<a$, ensures that the shadow is not the whole ground), and as pointed out above, it is amenable. In order to conclude the proof, we show that for all $s>m$, 
$s \notin \mathrm{D}(m+q a+q b)$, if the set $\mathrm{D}(m+q a+q b) \cup\{s\}$ is amenable, then its shadow is larger than $\{m, m+1, \ldots, m+q b\}$. Write $s=m+u a+v$, with $0 \leq v<a$. If $u$ is zero, as $s \notin \mathrm{D}(m+q a+q b)$, we obtain that $v>q b$, obtaining in this way a new element in the shadow. So $u$ must be positive. We distinguish two cases.

- If $v \leq q b$, then as $s \notin \mathrm{D}(m+q a+q b)$, by the preceding remark, we deduce that $u$ must be greater than $\frac{v}{b}$. But then $m+u a+v-(m+a+b-1)=$ $(u-1) a+(v-b)+1$, and this element is in $S$ if and only if $v-b+1 \leq(u-1) b$ (Lemma 17), or equivalently, $v<u b$, which holds since $u>\frac{v}{b}$. This proves that $m+a+b-1$ is in the shadow of $\mathrm{D}(m+q a+q b) \cup\{s\}$ (under the assumption that this set is amenable), and it is not in $\{m, m+1, \ldots, m+q b\}$, a contradiction.

- Now assume that $v>q b$. Then the element $m+v$ is in the shadow of $\mathrm{D}(m+q a+q b) \cup\{s\}$, obtaining again a contradiction.

From an $(S, m, r)$-ordered amenable set, we can construct another $(S, m, r-1)$ ordered amenable set, just by removing its pivot.

Lemma 32. If $M$ is an ordered amenable set, whose shadow is not the whole ground, and $P$ is its pivot, then $M \backslash\{P\}$ is ordered amenable.

Proof. Observe that $P$ does not belong to $\mathrm{D}(M \backslash\{P\})$, and thus $M \backslash\{P\}$ is still amenable. From the definition of pivot, it follows easily that this set is also ordered amenable.

Let $r$ be a positive integer, then there exists $q \in \mathbb{Z}$ such that

$$
q+\frac{1}{2} b q(q-1) \leq r<1+q+\frac{1}{2} b q(q+1) .
$$

Define $\mathrm{h}(r)=q$. Thus we can write $r=\mathrm{h}(r)+\frac{1}{2} b \mathrm{~h}(r)(\mathrm{h}(r)-1)+s$, with $0 \leq s \leq$ $\mathrm{h}(r) b$. Hence

$$
r=\mathrm{h}(r)+\frac{1}{2} b \mathrm{~h}(r)(\mathrm{h}(r)-1)+k \mathrm{~h}(r)+j,
$$

with $-1 \leq k \leq b-1$ and $0<j \leq \mathrm{h}(r)(k=-1$ only in the case $r=\mathrm{h}(r)+$ $\frac{1}{2} b \mathrm{~h}(r)(\mathrm{h}(r)-1)$, and then $\left.j=\mathrm{h}(r)\right)$. Note that $\mathrm{h}(r)=0$ leads to $r=0$, so we may assume that $\mathrm{h}(r)>0$. Observe also that $j+k=0$ only when $\mathrm{h}(r)=1=j$ and $k=-1$.

Proposition 33. Let $r$ be a positive integer. Let $k$ and $j$ be as above. If $b(\mathrm{~h}(r)-$ $1)+k+1<a+b-1$, then the set

$$
\begin{aligned}
&(\mathrm{D}(m+(\mathrm{h}(r)-1)(a+b)) \cap[m, \infty)) \\
& \cup\{m+u a+v \mid(\mathrm{h}(r)-1) b+1 \leq v \leq(\mathrm{h}(r)-1) b+k, 0 \leq u \leq \mathrm{h}(r)-1\} \\
& \cup\{m+((\mathrm{h}(r)-1) b+k+1) a+v \mid 0 \leq v<j\}
\end{aligned}
$$

is an $r$-ordered amenable set.

Proof. This set is obtained from $\mathrm{D}(m+\mathrm{h}(r)(a+b))$ by repeating Lemma $321+$ $\mathrm{h}(r)+\frac{b}{2} \mathrm{~h}(r)(\mathrm{h}(r)+1)-r$ times.

Next we prove that ordered $(S, m, r)$-amenable sets have minimal shadow in the set of all $(S, m, r)$-amenable sets with shadow an interval containing $m$. As a consequence any two $(S, m, r)$-ordered amenable sets have the same shadow. 
Proposition 34. Let $M$ be an ordered $(S, m, r)$-amenable subset of $S$ whose shadow has $t$ elements and let $N$ be another $(S, m, r)$-amenable subset of $S$ whose shadow is an interval containing $m$. Then, the shadow of $N$ has at least $t$ elements.

Proof. Suppose that the shadow of $N$ has less than $t$ elements. This implies that the set $N \backslash M$ is non-empty (since both sets have cardinality $r$ ) and therefore it has a minimum $z$. Furthermore, $N$ has no elements in the wagon of $M$. It is straightforward to observe that $M \cup\{z\}$ is amenable.

In fact, as $N$ is amenable, we have that $\mathrm{D}(z) \cap[m, \infty) \subset N ;(\mathrm{D}(z) \backslash\{z\}) \cap[m, \infty) \subset$ $M$ because $z$ is minimum. So $\mathrm{D}(z) \cap[m, \infty) \subset M \cup\{z\}$. As $z$ is not in the column containing the wagon of $M$ we conclude that $z \neq P+\rho_{2}$, which contradicts the assumption that $M$ is ordered.

Considering $M$ and $N$ ordered amenable sets in the above proposition and applying it in both directions, we get the following consequence.

Corollary 35. The shadows of ordered $(S, m, r)$-amenable sets coincide.

With all of these ingredients we can effectively compute the cardinality of the shadow of an ordered $(S, m, r)$-amenable set.

Corollary 36. Let $M$ be an ordered $(S, m, r)$-amenable set, and let $k$ and $j$ be as in (4), then $\#(M \cap[m, m+a+b))=(\mathrm{h}(r)-1) b+k+2$.

Proof. As any two ordered amenable sets with the same cardinality have the same elements in the ground, we can use the ordered ameneable set of the preceding proposition. Observe that the ground for this set is $\{m, m+1, \ldots, m+(\mathrm{h}(r)-$ 1) $b, m+(\mathrm{h}(r)-1) b+1, \ldots, m+(\mathrm{h}(r)-1)+k+1\}$.

Observe that this result gives a bound for integers $r$ such that there exists an ordered $(S, m, r)$-amenable set.

Now we prove that if $M$ is an $(S, m, r)$-amenable set whose shadow is not an interval containing $m$, then we can remove the trailing spaces in the shadow without increasing the number of divisors, that is, we can find $N$, an $(S, m, r)$-amenable with shadow an interval containing $m$, such that $\sharp \mathrm{D}(N) \leq \sharp \mathrm{D}(M)$. By using what we already know for ordered $(S, m, r)$-amenable sets, as a consequence we will obtain that they are optimal configurations. To this end we need several tools.

The first one enables us to push an $(S, m, r)$-amenable set to the right, obtaining an $(S, m+1, r)$-amenable set.

Lemma 37. Let $M$ be an $(S, m, r)$-amenable set. Then $N=M+1=\{x+1 \mid x \in$ $M\}$ is an $(S, m+1, r)$-amenable set.

Proof. Let $x=y+1 \in N$, with $y \in M$, and suppose that $h \in S$ is such that the divisor $x-h$ of $x$ is greater than $m$. We have to prove that $x-h \in N$. As $y-h$ is greater than or equal to $m$, we have that $y-h \in M$. It follows that $x-h=(y+1)-h=(y-h)+1 \in M+1=N$.

If we shift an $(S, m, r)$-amenable set to the left, we get an $(S, m-1, r)$-amenable set (provided $m-1 \geq 2 c-1$ ).

Lemma 38. Let $M$ be an $(S, m, r)$-amenable set with $m \geq 2 c$. Then $M-1=$ $\{x-1 \mid x \in M\}$ is an $(S, m-1, r)$-amenable set. 
Proof. Let $y \in M-1$, say, $y=x-1$, with $x \in M$. Note that $y \geq m$. Now we use Corollary 6. By hypothesis $\mathrm{D}(x) \cap[m, \infty)=(x-S) \cap[m, \infty) \subseteq M$, but then $\mathrm{D}(y) \cap[m, \infty)=((x-1)-S) \cap[m, \infty) \subseteq(M-1)$.

If we add the element $m$ to an $(S, m+1, r)$-amenable set, we get an $(S, m, r+1)$ amenable set.

Lemma 39. Let $N$ be an $(S, m+1, r)$-amenable set. The set $M=\{m\} \cup N$ is $(S, m, r+1)$-amenable.

Proof. If $N=\left\{m+1=m_{2}<\cdots<m_{r+1}\right\}$, then $M=\left\{m<m_{2}<\ldots<m_{r+1}\right\}$. Write $m_{1}=m$.

We have to check that $M$ is $m$-closed under division. It clearly holds for $i=1$. Let $i \geq 2$. The divisors of $m_{i}$ not smaller than $m+1$ belong to $N$ and thus to $M$. Therefore, divisors of $m_{i}$ not smaller than $m$ belong to $M$.

It is immediate that if we remove the biggest element of an $(m, r)$-amenable set, then we get an $(m, r-1)$-amenable set (provided $r>1)$.

Lemma 40. Let $M$ be an $(m, r)$-amenable set and suppose that $r>1$. Let $u$ be the maximum of $M$. Then $M \backslash\{u\}$ is an $(m, r-1)$-amenable set.

Before removing the trailing spaces of an $(S, m, r)$-amenable set, we need to see that it does not contain the last element in the ground, that is, $m+a+b-1$. If this is the case, then we give a procedure to obtain another $(S, m, r)$-amenable set whose shadow is at most as large as the original set, but not containing $m+a+b-1$.

Proposition 41. Given an $(S, m, r)$-amenable set $M$ with shadow $L_{M}$ not coinciding with the ground, we can construct an $(S, m, r)$-amenable set $N$ with shadow $L_{N}$ not containing $m+a+b-1$ and such that $\sharp L_{N} \leq \sharp L_{M}$.

Proof. Assume that $M$ is an $(S, m, r)$-amenable set containing $m+a+b-1$, and with a shadow different to the ground. Then, there is at least an element $x$ in the ground, such that $x$ is not in $M$, and thus $x<m+a+b-1$. Let $N=$ $\{m\} \cup(M+1) \backslash\{\max \{M+1\}\}$. The set $N$ is by the preceding lemmas an $(S, m, r)$ amenable set. Observe also that $x+1 \notin N$. Hence we repeat this procedure until $x+k$ becomes $m+a+b-1$.

Suppose we have a configuration not containing $m+a+b-1$. We can shrink it so that the shadow of the configuration obtained is an interval containing $m$. It can be done using the following results.

Lemma 42. Let $M$ be an $(S, m, r)$-amenable set not containing $m+a+b-1$. Assume there exists a column $c$, such that $c \cap M=\emptyset$, and if $M_{1}$ are the elements in $M$ in the columns to the left of $c$, and $M_{2}=M \backslash M_{1}$, then $M_{2} \neq \emptyset$ (c is a splitting column for $M)$. Let $r_{1}=\sharp M_{1}, r_{2}=\sharp M_{2}$, and $m_{2}=\min M_{2}$. Then

(1) $M_{1}$ is an $\left(S, m, r_{1}\right)$ amenable set,

(2) $M_{2}$ is an $\left(S, m_{2}, r_{2}\right)$ amenable set,

(3) $M_{1} \cup\left(M_{2}-1\right)$ is an $(S, m, r)$ amenable set.

Proof. It suffices to show that no element in $M_{1}$ divides an element in $M_{2}$, and vice-versa. Assume that there is $x \in M_{1}$ and $y \in M_{2}$ such that $y-x \in S$, that is, $y-x=k a+r$ for some $r, k$ non-negative integers with $r \leq \min \{a-1, k b\}$ (Lemma 17). Hence $y=x+k a+r$, and $y-(k a+i) \in \mathrm{D}(M) \cap[m, \infty)=M$ for all 
$i \in\{0, \ldots, r\}$. Assume that $c$ corresponds with the elements $s$ in $[m, \infty) \cap \mathbb{N}$ such that $s-(m+b) \bmod a=j$. Then, by hypothesis, we get

$$
\begin{aligned}
& y-r-(m+b) \bmod a=y-(k a+r)-(m+b) \bmod a \\
& \quad=x-(m+b) \bmod a<y-(m+b) \bmod a,
\end{aligned}
$$

and thus there is $i \in\{0, \ldots, r\}$ such that $y-(k a+i)-(m+b) \bmod a=y-i-$ $(m+b) \bmod a=j$, contradicting that $c$ was an empty column of $M$.

Now assume that $x \in M_{1}$ and $y \in M_{2}$ are such that $x-y=k a+r$ for some $r, k$ as above. In this setting, $x-(k a+i) \in M$ for all $i \in\{0, \ldots, r\}$. By hypothesis $x-(m+b) \bmod a<y-(m+b) \bmod a$, and

$$
\begin{aligned}
y+r-(m+b) \bmod a=y+k a+ & -(m+b) \bmod a \\
& =x-(m+b) \bmod a<y-(m+b) \bmod a .
\end{aligned}
$$

It follows that for some $i \in\{0, \ldots, r\}, y+i-(m+b) \bmod a=a-1$, but this is impossible, since as $m+a+b-1 \notin M$, the column $\{s \in M \mid s-(m+b) \bmod a=a-1\}$ is empty.

Proposition 43. Let $M$ be an $(S, m, r)$-amenable set whose shadow $L_{M}$ has $t$ elements. There exists an $(S, m, r)$-amenable set $T$ whose shadow $L_{T}$ is an interval containing $m$ and has no more than $t$ elements, i.e., $\sharp L_{T} \leq \sharp L_{M}$.

Proof. Every time you find a splitting column as in the statement of Lemma 42 , change $M$ with $M_{1} \cup\left(M_{2}-1\right)$. This procedure does not increase the number of elements in the shadow of $M$.

Lemma 44. Let $M$ be an $(S, m, r)$-amenable set whose shadow $L_{M}$ has $t$ elements. There exists an $(S, m, r)$-amenable set $T$ whose shadow is an interval containing $m$, and $\sharp \mathrm{D}(T) \leq \sharp \mathrm{D}(M)$.

Proof. Let $T$ be as in Proposition 43 , and assume that $\sharp L_{T}=t-k$. In view of Proposition 28, $\sharp \mathrm{D}\left(L_{M}\right) \geq \sharp \mathrm{D}(m, m+1, \ldots, m+t-1)$. By Corollary 23, $\sharp \mathrm{D}(m, m+$ $1, \ldots, m+t-1)=\sharp \mathrm{D}(m)+\sum_{j=1}^{t-1}\left\lceil\frac{a+b-j}{b}\right\rceil$, and as $\left\lceil\frac{a+b-j}{b}\right\rceil \geq 1$, this amount is greater than or equal to $\sharp \mathrm{D}(m)+\sum_{j=1}^{t-k-1}\left[\frac{a+b-j}{b}\right]+k$, which according to Corollary 23 equals $\sharp \mathrm{D}(m, m+1, \ldots, m+t-k-1)+k=\sharp \mathrm{D}\left(L_{T}\right)+k$. Now we use Lemma 13. having $\sharp \mathrm{D}(M)=\sharp \mathrm{D}\left(L_{M}\right)+\sharp M \backslash L_{M}=\sharp \mathrm{D}\left(L_{M}\right)+r-t \geq \sharp \mathrm{D}\left(L_{T}\right)+k+r-t=$ $\sharp \mathrm{D}\left(L_{T}\right)+\sharp T \backslash L_{T}=\sharp \mathrm{D}(T)$.

Theorem 45. Let $S$ be a numerical semigroup with conductor $c$, and let $m \geq 2 c-1$. Then every ordered $(S, m, r)$-amenable set is an optimal configuration.

Proof. Let $M$ be an ordered $(S, m, r)$-amenable set. By Proposition 10, among the optimal configurations, there is always an $(S, m, r)$-amenable set. Let $N$ be an $(S, m, r)$-amenable set that is an optimal configuration. In light of Lemma 44, we can assume that its shadow is an interval containing $m$. By Proposition [34, the shadow of $M$ is contained in that of $N$, and by Corollary 14 we get that $\# \mathrm{D}(M) \leq$ $\# \mathrm{D}(N)$. As $N$ is an optimal configuration we deduce that $\# \mathrm{D}(M)=\# \mathrm{D}(N)$, and thus $M$ is also an optimal configuration.

Corollary 46. Let $S=\langle a, a+1, \ldots, a+b\rangle$ with integers $a, b$ such that $0<b<a$. Write $r$ as in formula (4), that is, $r=\mathrm{h}(r)+\frac{1}{2} b \mathrm{~h}(r)(\mathrm{h}(r)-1)+k \mathrm{~h}(r)+j$, with 
$-1 \leq k \leq b-1$ and $0<j \leq \mathrm{h}(r)$. Then the $r$-th Feng-Rao Number of $S$ (see Definition (4), $\mathrm{E}(\langle a, a+1, \ldots, a+b\rangle, r)$, equals

$$
r-1+ \begin{cases}\sum_{i=1}^{b(\mathrm{~h}(r)-1)+k+1}\left\lceil\frac{a-i}{b}\right\rceil, & \text { if } b(\mathrm{~h}(r)-1)+k+2<a+b, \\ \sum_{i=1}^{a+b-1}\left\lceil\frac{a-i}{b}\right\rceil, & \text { otherwise. }\end{cases}
$$

Proof. Assume that $b(\mathrm{~h}(r)-1)+k+1<a+b-1$. Then by Proposition 33, there exists an ordered $(S, m, r)$-amenable set. In this setting the proof follows from Corollaries 24 and 36 and Theorem 45.

Observe also that if $b(\mathrm{~h}(r)-1)+k+1=a+b-2$, by Proposition 33, the set

$$
\begin{aligned}
& M=(\mathrm{D}(m+(\mathrm{h}(r)-1)(a+b)) \cap[m, \infty)) \\
& \quad \cup\{m+u a+v \mid(\mathrm{h}(r)-1) b+1 \leq v \leq(\mathrm{h}(r)-1) b+k+1,0 \leq u \leq \mathrm{h}(r)-1\}
\end{aligned}
$$

is an ordered amenable set, and thus by Theorem 45 an optimal configuration for $r=\# M$. As $\mathrm{D}(M \cup\{m+a+b-1\})=\mathrm{D}(M) \cup\{m+a+b-1\}$, the set $M \cup\{m+a+b-1\}$ is an optimal configuration of cardinality $r+1$, whose shadow fills the whole ground. Now by using Corollary [16, we get optimal configurations for cardinalities greater than $r+1$; the proof follows easily by Corollary 24.

Needless to say that, by using this formula for numerical semigroups generated by intervals, we have no need of the general Algorithm 3 speeding-up the computation of Feng-Rao distances for such semigroups.

Remark 47. The reader can check that we have $\mathrm{E}(S, r)=\rho_{r}$ exactly in the following cases:

(A) if either $r=b \sigma(p)+1, \ldots, b \sigma(p)+p+1$ and the ground is not completely filled, or

(B) $r \geq r_{0}$, where $r_{0}$ is the first $r$ filling the ground, being $\sigma(p):=1+\cdots+p=$ $\frac{1}{2} p(p+1)$ and $p \geq 1$.

Therefore, since both sequences $\mathrm{E}(S, r)$ and $\rho_{r}$ are strictly increasing, the largest difference between them is for $r=2$ and for the first $r$ after each element in the first case, that is, $r=b \sigma(p)+p+2$ where $\rho_{r}$ jumps from one interval to the next.

\section{ACKNOWLEDGEMENT}

The authors would like to thank the referees for their comments and suggestions.

\section{REFERENCES}

[1] A. Barbero and C. Munuera, "The weight hierarchy of Hermitian codes", SIAM J. Discrete Math. vol. 13, no. 1, pp. 79-104 (2000). MR1737936 (2001g:94021)

[2] A. Campillo and J. I. Farrán, "Computing Weierstrass semigroups and the Feng-Rao distance from singular plane models", Finite Fields and their Applications 6, pp. 71-92 (2000). MR 1738217 (2001a:14026)

[3] A. Campillo, J. I. Farrán and C. Munuera, "On the parameters of algebraic geometry codes related to Arf semigroups", IEEE Trans. of Information Theory 46, pp. 2634-2638 (2000). MR $1806823(2001 \mathrm{k}: 94073)$

[4] S. T. Chapman, P. A. García-Sánchez and D. Llena, "The catenary and tame degree of a numerical semigroup", Forum Math. 21, pp. 117-129 (2009). MR2494887 (2010i:20081)

[5] M. Delgado, P. A. García-Sánchez and J. Morais, "NumericalSgps", A GAP package for numerical semigroups, current version number 0.97 (2011). Available via http: //www.gap-system.org/.

[6] J. I. Farrán, P. A. García-Sánchez and D. Llena, "On the Feng-Rao numbers", Actas de las VII Jornadas de Matemática Discreta y Algorítmica, pp. 321-333 (2010). 
[7] J. I. Farrán and C. Munuera, "Goppa-like bounds for the generalized Feng-Rao distances", Discrete Applied Mathematics 128/1, pp. 145-156 (2003). MR 1991422(2004f:94115)

[8] G. L. Feng and T. R. N. Rao, "Decoding algebraic-geometric codes up to the designed minimum distance", IEEE Trans. Inform. Theory 39, pp. 37-45 (1993). MR 1211489 (93m:94031)

[9] P. Heijnen and R. Pellikaan, "Generalized Hamming weights of $q$-ary Reed-Muller codes", IEEE Trans. Inform. Theory 44, pp. 181-197 (1998). MR.1486657(99a:94068)

[10] T. Helleseth, T. Kløve and J. Mykkleveit, "The weight distribution of irreducible cyclic codes with block lengths $n_{1}\left(\left(q^{l}-1\right) / N\right)$ ", Discrete Math., vol. 18, pp. 179-211 (1977). MR.0446717 (56:5041)

[11] T. Høholdt, J. H. van Lint and R. Pellikaan, "Algebraic Geometry codes", in Handbook of Coding Theory, V. Pless, W.C. Huffman and R.A. Brualdi, Eds., pp. 871-961 (vol. 1), Elsevier, Amsterdam (1998). MR1667946

[12] C. Kirfel and R. Pellikaan, "The minimum distance of codes in an array coming from telescopic semigroups", IEEE Trans. Inform. Theory 41, pp. 1720-1732 (1995). MR1391031 (97e:94015)

[13] J. C. Rosales and P. A. García-Sánchez, "Numerical Semigroups", Developments in Maths. vol. 20, Springer (2010).

[14] V. Wei, "Generalized Hamming weights for linear codes", IEEE Trans. Inform. Theory 37, pp. 1412-1428 (1991). MR.1136673(92i:94019)

CMup, Departamento de Matematica, Faculdade de Ciencias, Universidade do Porto, Rua do Campo Alegre 687, 4169-007 Porto, Portugal

E-mail address: mdelgado@fc.up.pt

Departamento de Matemática Aplicada, Escuela Universitaria de Informática, CamPus de Segovia - Universidad de Valladolid, Plaza de Santa Eulalia 9 y 11 - 40005 SEgOvia, Spain

E-mail address: jifarran@eii.uva.es

Departamento de Álgebra, Universidad de Granada, 18071 Granada, España

E-mail address: pedro@ugr.es

Departamento de Geometría, Topología y Química Orgánica, Universidad de Almería, 04120 Almería, España

E-mail address: dllena@ual.es 